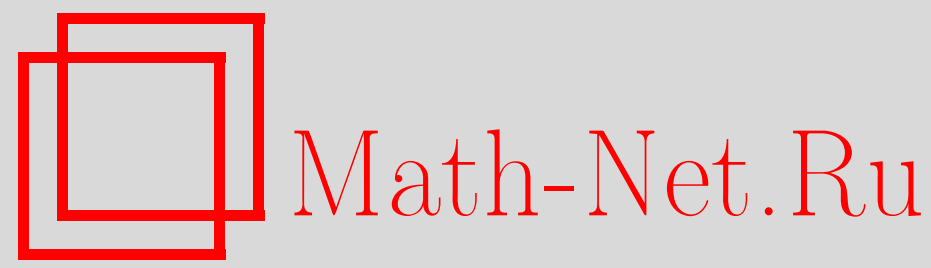

А. И. Ермаков, Наилучшие хаусдорфовы приближения алгебраическими полиномами и пористость, Матем. заметки, 1996, том 59, выпуск $5,692-702$

DOI: https://doi.org/10.4213/mzm1763

Использование Общероссийского математического портала MathNet.Ru подразумевает, что вы прочитали и согласны с пользовательским соглашением

http://www . mathnet.ru/rus/agreement

Параметры загрузки:

IP : 54.198 .67 .100

26 апреля 2023 г., 14:24:52

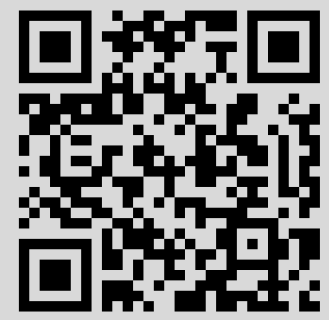


НАИЛУЧШИЕ ХАУСДОРФОВЫ ПРИБЛИЖЕНИЯ АЛГЕБРАИЧЕСКИМИ ПОЛИНОМАМИ

\section{И ПОРИСТОСТЬ}

\section{А. И. Ермаков}

Определения и обозначения, которьми будем пользоваться ниже, приведены в [1] и [2]. Напомним, что хаусдорфовым расстоянием между двумя множествами $E_{1}$ и $E_{2}$ метрического пространства $(X, \rho)$ назьвается величина

$$
H_{\rho}\left(E_{1}, E_{2}\right)=\inf \left\{\varepsilon \mid E_{1} \subset U_{\varepsilon}\left(E_{2}\right), E_{2} \subset U_{\varepsilon}\left(E_{1}\right)\right\},
$$

где $U_{\varepsilon}(\cdot)-\varepsilon$-окрестность множества относительно метрики $\rho$. При $\alpha>0$ $\alpha$-метрика Хаусдорфа $H_{\alpha}$ - это метрика, порожденная $\alpha$-метрикой Минковского на плоскости $x O y$ :

$$
\rho_{\alpha}\left(\left(x_{1}, y_{1}\right),\left(x_{2}, y_{2}\right)\right)=\max \left\{\frac{\left|x_{1}-x_{2}\right|}{\alpha},\left|y_{1}-y_{2}\right|\right\} \text {. }
$$

Дополненньй график $F(f)$ ограниченной, вообще говоря многозначной, функции $f$ вешественной переменной определяется как наименьшее замкнутое множество плоскости $x O y$, содержащее график $\{(x, f(x))\}$ этой функции и вместе с каждой парой точек $\left(x, y_{1}\right),\left(x, y_{2}\right)$, содержащее вертикальный отрезок с концами в этих точках. Нам удобно считать, что график функции $f$ совпадает с ее дополненным графиком $F(f)$. Хаусдорфовым $\alpha$-расстоянием между ограниченными функциями $f$ и $g$, заданными на множестве $E \subset \mathbb{R}$, назьвается хаусдорфово $\alpha$-расстояние между их дополненными графиками: $H_{\alpha}(f, g, E)=H_{\alpha}(f, g)=H_{\alpha}(F(f), F(g))$. Если $\Delta=[a, b], 0<\varepsilon<|b-a| / 2, f_{\varepsilon}-$ сужение функции $f(\operatorname{dom} f=\Delta)$ на отрезок $[a+\varepsilon, b-\varepsilon]$, то

$$
\widetilde{H}_{\alpha}(f, g, \Delta) \stackrel{\text { def }}{=} \inf \left\{\varepsilon \mid F\left(f_{\varepsilon}\right) \subset U_{\varepsilon}(F(g)), F\left(g_{\varepsilon}\right) \subset U_{\varepsilon}(F(f))\right\} .
$$

Работа выполнена при поддержке Российского фонда фундаментальных исследований, грант № 93-01-00236. 
Через $H_{\alpha} E_{n}(f, \Delta)$ обозначают наименьшее уклонение заданной на $\Delta$ функции $f$ от алгебраических полиномов степени не большей $n$, через $H_{\alpha} E_{n}(f)$ - наименьшее уклонение $2 \pi$-периодической функции $f$ от тригонометрических полиномов порядка не большего $n$ в $\alpha$-метрике Хаусдорфа. Положим $\Im=[-1,1], c_{\alpha}(f, \Im)=\underline{\lim } n H_{\alpha} E_{n}(f, \Im), c_{\alpha}(f)=$ $\underline{\lim } n H_{\alpha} E_{n}(f)$. Изучение величин $c_{\alpha}(f)$ и $c_{\alpha}(f, \Im)$ положило начало исследованиям, связанным с “эффектом констант", неожиданно обнаруженным Е.П. Долженко и Е.А. Севастьяновьм [3]. Эти величины позволяют описать ряд свойств функции (см. [2], [4]). Так, если $c_{\alpha}(f)<\pi / \alpha$, то $2 \pi$-периодическая функция $f$ однозначна и непрерывна почти всюду, если $c_{\alpha}(f)<\pi /(2 \alpha)$ - всюду. С другой стороны, для любого $\alpha>0$ сушествуют всюду разрывная функция $g$, для которой $c_{\alpha}(g)=\pi / \alpha$, и разрьвная функция $\varphi$, для которой $c_{\alpha}(\varphi)=\pi /(2 \alpha)$. Сходные результаты получены и в случае приближения функции алгебраическими полиномами [5]. При $c_{\alpha}(f, \Im) \geqslant \pi / \alpha$ нельзя гарантировать однозначности и непрерывности функции $f$ ни в одной точке, на что указьвает пример функции с $c_{\alpha}(f, \Im)=\pi / \alpha$, график которой есть квадрат $\{(x, y) \mid-1 \leqslant x, y \leqslant 1\}$. Если $c_{\alpha}(f, \Im)=\pi \sqrt{1-\left(a^{\prime}\right)^{2}} / \alpha, 0<a^{\prime} \leqslant 1$, то $f$ однозначна и непрерьвна почти всюду на отрезке $\left[-a^{\prime}, a^{\prime}\right]$, а если $c_{\alpha}(f, \Im)=\pi \sqrt{1-a^{2}} /(2 \alpha), 0<\alpha \leqslant 1$, то $f$ непрерывна на интервале $(-a, a)$. При этом для любого положительного $a^{\prime}<1$ найдется заданная на $\Im$ функция $h$, непрерывная почти всюду на $\left[-a^{\prime}, a^{\prime}\right]$ и разрывная в остальных точках отрезка $\Im$, для которой $c_{\alpha}(h, \Im)=\pi \sqrt{1-\left(a^{\prime}\right)^{2}} / \alpha$; если $0<\alpha \leqslant 1$, то найдется заданная на $\Im$ функция $\psi$ с разрьвами второго рода в точках $\pm a$, для которой $c_{\alpha}(\psi, \Im)=\pi \sqrt{1-a^{2}} /(2 \alpha)$. Для меры множества $D(f)$ точек разрыва функции $f$, заданной на $\Im$, при $0<c_{\alpha}(f, \Im)<\pi / \alpha$ имеет место [5] оценка

$$
\operatorname{mes} D(f) \leqslant 2 d=2\left(1-\sqrt{1-\alpha^{2} c_{\alpha}^{2}(f, \Im) \pi^{-2}}\right)<2 c_{\alpha}(f, \Im) .
$$

Причем множество $D(f)$ с точностью до подмножества меры 0 находится на множестве $[-1,-1+d] \cup[1-d, 1]$. В случае $0<c_{\alpha}(f, \Im)<\pi /(2 \alpha)$ множество $D(f)$ целиком находится на множестве $\left[-1,-1+d^{\prime}\right] \cup\left[1-d^{\prime}, 1\right]$, где $2 d^{\prime}=2\left(1-\sqrt{1-4 \alpha^{2} c_{\alpha}^{2}(f, \Im) \pi^{-2}}\right)<2 \alpha c_{\alpha}(f, \Im)$. А. П. Петуховым [6] изучена зависимость свойств множества $D(f)$ точек разрыва $2 \pi$-периодической функции $f$ от величины $c_{\alpha}(f)$. Характеристикой множества $D(f)$, связанной с $c_{\alpha}(f)$, оказался коэффициент $\sigma$-равнопористости множества. Само понятие пористости было введено в анализ Е. П. Долженко [7] для характеризации граничных свойств функций. Пусть $E$-множество в метрическом пространстве $(X, \rho), x \in X, r(\varepsilon, x, E)$ - точная верхняя грань радиусов шаров из $\varepsilon$-окрестности точки $x$, не пересекаюшихся с множеством $E$. Величину

$$
r_{0}(E)=\varlimsup_{\varepsilon \rightarrow 0} \inf _{x \in E} \frac{r(\varepsilon, x, E)}{\varepsilon}
$$


назовем коэффищиентом равнопористости множества $E$, а множество $E_{0}$, для которого $r_{0}\left(E_{0}\right)>0,-$ равнопористым. Множество $E$, представимое в виде объединения счетного числа множеств $\left\{E_{i}\right\}_{i=1}^{\infty}$, для которых $\inf r_{0}\left(E_{i}\right) \geqslant a>0$, назовем $\sigma$-равнопористым, а величину

$$
R(E) \stackrel{\text { def }}{=} \sup _{\cup E_{i} \supset E}\left\{\inf r_{0}\left(E_{i}\right)\right\}
$$

где sup берется по всем не более чем счетным покрытиям множества $E,-$ коэффициентом $\sigma$-равнопористости множества $E$. Положим $a(c)=$ $\sqrt{1-(2 \alpha c / \pi)^{2}}$, если $0<c \leqslant \pi /(2 \alpha)$, и $a(c)=0$, если $\pi /(2 \alpha)<c<\pi / \alpha$; $a^{\prime}(c)=\sqrt{1-(\alpha c / \pi)^{2}}$. В алгебраическом случае имеют место следующие утверждения.

ТЕОРЕМА 1. Пусть $f$ - ограниченная (вообще говоря, многозначная) функиия, заданная на отрезке $\Im, 0<c=c_{\alpha}(f, \Im)<\pi / \alpha$. Тогда при любом $b \in\left(a(c), a^{\prime}(c)\right]$ имеет место оиенка

$$
R(D(f) \cap[-b, b]) \geqslant \frac{2 \pi \sqrt{1-b^{2}}-2 \alpha c_{\alpha}(f, \Im)}{3 \pi \sqrt{1-b^{2}}-2 \alpha c_{\alpha}(f, \Im)}
$$

ТЕОрема 2. Для любого $\alpha>0$, любого $c_{0}$, удовлетворяюшего неравенству $0<c_{0}<\pi / \alpha, u$ любого $b \in\left(a\left(c_{0}\right), a^{\prime}\left(c_{0}\right)\right]$ существует заданная на отрезке $\Im$ функиия $g$, для которой $c_{\alpha}(g, \Im)=c_{0} u$

$$
R(D(g) \cap[-b, b])=\frac{2 \pi \sqrt{1-b^{2}}-2 \alpha c_{0}}{3 \pi \sqrt{1-b^{2}}-2 \alpha c_{0}} .
$$

ДОКАЗАТЕЛЬСТВО ТЕОРЕМЫ 1 вытекает из следующих двух лемм.

ЛЕмма 1 (см. [5]). Если $\underline{\lim } n H_{\alpha} E_{n}(f, \Im) \leqslant c, P_{n}$ - алгебраический полином степени не большей $n$ наилучшего приближсени функиии $f$ в $\alpha$-метрике Хаусдорфа, $f^{*}(t)=f(\cos t), P_{n}^{*}(t)=P_{n}(\cos t)(0 \leqslant t \leqslant \pi)$ $u 0<b<1$, mo

$$
\underline{\lim } n \widetilde{H}_{\alpha}\left(f^{*}, P_{n}^{*},[\arccos b, \pi-\arccos b]\right) \leqslant c\left(1-b^{2}\right)^{-1 / 2} .
$$

ЛЕмма 2. Пусть функиия $f$ задана и, вообще говоря, неоднозначна на отрезке $\Delta \subset[-\pi, \pi], a\left\{T_{n}\right\}_{n=1}^{\infty}-$ последовательность тригонометрических полиномов степени не большей $n$ соответственно, в совокупности по абсолютной величине ограниченных на $(-\infty, \infty)$ некоторой постоянной $\mathscr{K}$. Если

$$
\frac{\pi}{2 \alpha} \leqslant \widetilde{c}_{\alpha}(f, \Delta) \stackrel{\text { def }}{=} \underline{\lim } n \widetilde{H}_{\alpha}\left(f, T_{n}, \Delta\right) \leqslant \frac{\pi}{\alpha},
$$


mo

$$
R(D(f)) \geqslant \frac{2 \pi-2 \alpha \widetilde{c}_{\alpha}(f, \Delta)}{3 \pi-2 \alpha \widetilde{c}_{\alpha}(f, \Delta)} .
$$

Эта лемма по формулировке и доказательству аналогична теореме 1 работы [6], где функция $f$ задана на промежутке $(-\infty, \infty)$. Отметим, что если $\widetilde{c}_{\alpha}(f, \Delta)<\pi /(2 \alpha)$ и выполнены остальные условия леммы 2 , то функция $f$ непрерывна внутри $\Delta$.

Полагая в лемме $1 c=c_{\alpha}(f, \Im)$, получаем

$$
\underline{\lim } n \widetilde{H}_{\alpha}\left(f^{*}, P_{n}^{*},[\arccos b, \pi-\arccos b]\right) \leqslant c\left(1-b^{2}\right)^{-1 / 2} .
$$

Так как $b \in\left(a(c), a^{\prime}(c)\right]$, то $\pi /(2 \alpha)<c_{\alpha}(f, \Im)\left(1-b^{2}\right)^{-1 / 2} \leqslant \pi / \alpha$. Множество полиномов $\left\{P_{n}\right\}_{n=1}^{\infty}$, а, следовательно, и множество тригонометрических полиномов $\left\{P_{n}^{*}\right\}_{n=1}^{\infty}$ равномерно ограничены по модулю соответственно на отрезке $\Im$ и на прямой $(-\infty, \infty)$ константой $\mathscr{K}=2 \sup \{|f(x)| \mid x \in \Im\}$. Применяя к функции $f^{*}$, заданной на отрезке $\Delta=[\arccos b, \pi-\arccos b]$, лемму 2 , получаем, что

$$
R\left(D\left(f^{*}\right)\right) \geqslant \frac{2 \pi \sqrt{1-b^{2}}-2 \alpha c_{\alpha}(f, \Im)}{3 \pi \sqrt{1-b^{2}}-2 \alpha c_{\alpha}(f, \Im)} .
$$

В силу локальной линейности отображения $x=\cos t(0 \leqslant t \leqslant \pi)$, тогда и

$$
R(D(f) \cap[-b, b]) \geqslant \frac{2 \pi \sqrt{1-b^{2}}-2 \alpha c_{\alpha}(f, \Im)}{3 \pi-2 \alpha c_{\alpha}(f, \Im)},
$$

что доказьвает теорему 1.

ДОКАЗАТЕЛЬСТВО ТЕОРЕМЫ 2. Основой доказательства ЯВляется

ЛЕмма 3. Для любого $\alpha>0$, любого $\widetilde{c}_{0} \in(\pi /(2 \alpha), \pi / \alpha)$ и любой возрастающей последовательности натуральных чисел существуют $2 \pi$-периодическая функция $f_{0} c \sup _{t \in \mathbb{R}}|f(t)|=1$ и последовательность тригонометрических полиномов $\left\{T_{n_{i}}\right\}_{i=1}^{\infty}$ такие, что

$$
\begin{aligned}
& H_{\alpha}\left(f_{0}, T_{n_{i}}\right) \leqslant \frac{\widetilde{c}_{0}\left(1+2^{-i+1}\right)}{n_{i}}, \quad i=1,2, \ldots ; \\
& R\left(D\left(f_{0}\right) \cap\left(-\frac{2 \alpha \widetilde{c}_{0}-\pi}{2 n_{1}}, \frac{2 \alpha \widetilde{c}_{0}-\pi}{2 n_{1}}\right)\right) \leqslant \frac{2 \pi-2 \alpha \widetilde{c}_{0}}{3 \pi-2 \alpha \widetilde{c}_{0}}
\end{aligned}
$$

3) $\left\{n_{i}\right\}_{i=1}^{\infty}-$ подпоследовательность заданной возрастающей последовательности натуральных чисел, первый член $n_{1}$ которой мохет быть взят сколь угодно больиим.

Конструкция функции $f_{0}$ и выбор полиномов $\left\{T_{n_{i}}\right\}_{i=1}^{\infty}$ описаны А. П. Петуховьм в работе [6, с. 983-987]. Построение А.П. Петухова таково, что 
начальное число $n_{1}$, положенное единице, может быть взято любым как угодно большим; каждое из определяемых по индукции чисел $n_{1}, n_{2}, \ldots$ подчинено только ограничениям снизу (см. [6, с. 984]) и может быть выбрано произвольным достаточно большим, в частности, принадлежашим заданной возрастающей последовательности натуральных чисел.

Положим $\rho=\left(2 \alpha \widetilde{c}_{0}-\pi\right) /\left(2 n_{1}\right), m_{i}=\left[\sqrt{n_{i}}+1\right]$. Нам понадобится обрашаюшаяся в нуль на множестве $[-\pi, \pi] \backslash[-\rho, \rho] 2 \pi$-периодическая четная функция $\psi \in \widetilde{c}^{(8)} \mathrm{c} \max _{t \in \mathbb{R}, 0 \leqslant k \leqslant 8}\left|\psi^{(k)}(t)\right| \leqslant M$, где значение положительной постоянной $M$ уточним позже. Пусть $W_{m_{i}}$ - тригонометрический полином наименьшего уклонения порядка $\leqslant m_{i}$ от (нечетной) функции $\psi^{\prime}$. Тогда согласно результату Ж. Фавара, Н.И. Ахиезера, М. Г. Крейна и теореме Н. П. Корнейчука имеем

$$
\left|\psi^{\prime}(t)-W_{m_{i}}(t)\right| \leqslant \frac{\pi \mathscr{K}_{6} M}{2\left(m_{i}+1\right)^{6}} \leqslant \frac{\pi \mathscr{K}_{6} M}{2 n_{i}^{3}}<\frac{2 M}{n_{i}^{3}},
$$

так как постоянная Фавара $\mathscr{K}_{6}<4 / \pi$, Отсюда следует, что для полинома $P_{m_{i}}(t) \stackrel{\text { def }}{=} \int_{-\pi}^{t} W_{m_{i}}(t) d t, t \in(-\infty, \infty)$, будет

$$
\left|\psi(t)-P_{m_{i}}(t)\right|<\frac{A}{n_{i}^{3}},
$$

где $A=4 \pi M$. В частности,

$$
\left|P_{m_{i}}(t)\right|<\frac{A}{n_{i}^{3}} \quad \text { при } t \in[-\pi, \pi] \backslash[-\rho, \rho] .
$$

Введем $2 \pi$-периодическую функцию $\varphi_{0}=f_{0} \psi$ и тригонометрические полиномы $\sigma_{r_{i}}=T_{n_{i}} P_{m_{i}}$, где $r_{i}=n_{i}+m_{i}$. Так как $D\left(\varphi_{0}\right) \cap(-\rho, \rho)=$ $D\left(f_{0}\right) \cap(-\rho, \rho)$, то $R\left(D\left(\varphi_{0}\right) \cap(-\rho, \rho)\right) \leqslant\left(2 \pi-2 \alpha \widetilde{c}_{0}\right) /\left(3 \pi-2 \alpha \widetilde{c}_{0}\right)$ в силу $(2)$. Очевидно, что

$$
\begin{array}{rcc}
\varphi_{0}(t)=0 & \text { при } & t \in[-\pi, \pi] \backslash[-\rho, \rho], \\
\left|\sigma_{r_{i}}(t)\right|<\frac{2 A}{n_{i}^{3}} & \text { при } & t \in[-\pi, \pi] \backslash[-\rho, \rho] .
\end{array}
$$

Обозначим через $\tau_{i}$ величину $\widetilde{c}_{0}\left(1+2^{-i+1}\right) / n_{i}$. Из неравенства (1) и определения хаусдорфова $\alpha$-расстояния между двумя функциями следует, что для любого $t$ и любого значения $f_{0}(t)$ функции $f_{0}$ найдется такое $t_{i}$, для которого $\left|t-t_{i}\right| \leqslant \alpha \tau_{i},\left|f_{0}(t)-T_{n_{i}}\left(t_{i}\right)\right| \leqslant \tau_{i}$. Отсюда и из (3)-(5) вытекает

$$
\begin{aligned}
\left|\varphi_{0}(t)-\sigma_{r_{i}}\left(t_{i}\right)\right| \leqslant & \left|f_{0}(t)\right| \cdot\left|\psi(t)-P_{m_{i}}(t)\right|+\left|P_{m_{i}}(t)\right| \cdot\left|f_{0}(t)-T_{n_{i}}(t)\right| \\
& +\left|T_{n_{i}}\left(t_{i}\right)\right| \cdot\left|W_{m_{i}}(\xi)\right| \cdot\left|t-t_{i}\right| \\
\leqslant & (M+2 \alpha M) \tau_{i}+\frac{A+A \tau_{i}+4 \alpha M \tau_{i}}{n_{i}^{3}}, \quad \xi \in\left(t, t_{i}\right) .
\end{aligned}
$$


Если считать, что $M=1 /(1+2 \alpha), B=(3 A+8 \alpha M) \widetilde{c}_{0}$, то окажется

$$
\left|\varphi_{0}(t)-\sigma_{r_{i}}\left(t_{i}\right)\right|<\tau_{i}+\frac{B}{n_{i}^{3}} .
$$

С другой стороны, для любого $t$ найдется такое $t_{i}^{\prime}$ и некоторое значение $f_{0}\left(t_{i}^{\prime}\right)$ функции $f_{0}$, что $\left|t-t_{i}^{\prime}\right| \leqslant \alpha \tau_{i},\left|f_{0}\left(t_{i}^{\prime}\right)-T_{n_{i}}(t)\right| \leqslant \tau_{i}$. Из этого и из (3)-(5) аналогично тому, как было получено неравенство (8), выводим неравенство

$$
\left|\varphi_{0}\left(t_{i}^{\prime}\right)-\sigma_{r_{i}}(t)\right|<\tau_{i}+\frac{B}{n_{i}^{3}}
$$

которое вместе с (8) дает

$$
H_{\alpha}\left(\varphi_{0}, \sigma_{r_{i}}\right) \leqslant \tau_{i}+\frac{B}{n_{i}^{3}} .
$$

Пусть теперь $2 \rho<\lambda_{k}<\pi / 2, \varphi_{k}(t)=\varphi_{0}\left(t-\lambda_{k}+\rho\right)+\varphi_{0}\left(-t-\lambda_{k}+\rho\right)$, $S_{r_{i}}(t)=\sigma_{r_{i}}\left(t-\lambda_{k}+\rho\right)+\sigma_{r_{i}}\left(-t-\lambda_{k}+\rho\right), t \in(-\infty, \infty)$. Отметим, что $\varphi_{k}$ и $S_{r_{i}}$ - четные $2 \pi$-периодические функции и $\max _{t \in \mathbb{R}}\left|\varphi_{k}(t)\right|=1$. Оценим величину $H_{\alpha}\left(\varphi_{0}, S_{r_{i}},[0, \pi]\right)$. С этой целью воспользуемся тем, что неравенство (9) остается верным, если у функций $\varphi_{0}$ и $\sigma_{r_{i}}$ аргумент $t$ заменить на $t-\lambda_{k}+\rho$ (такая замена означает параллельньй перенос графиков исходных функций $\varphi_{0}$ и $\sigma_{r_{i}}$, сохраняюший неизменньм хаусдорфово расстояние между ними). Но тогда для любого $t$ и любого значения $\varphi_{0}\left(t-\lambda_{k}+\rho\right)$ функции $\varphi_{0}$ найдется такое $\bar{t}_{i}$, что

$$
\left|t-\bar{t}_{i}\right| \leqslant \alpha \tau_{i}, \quad\left|\varphi_{0}\left(t-\lambda_{k}+\rho\right)-\sigma_{r_{i}}\left(\bar{t}_{i}-\lambda_{k}+\rho\right)\right| \leqslant \tau_{i}+\frac{B}{n_{i}^{3}} .
$$

Для любого $t$ найдется также такое $\bar{t}_{i}^{\prime}$ и некоторое значение $\varphi_{0}\left(\bar{t}_{i}^{\prime}-\lambda_{k}+\rho\right)$ функции $\varphi_{0}$, для которых $\left|t-\bar{t}_{i}^{\prime}\right| \leqslant \alpha \tau_{i}$ и $\left|\sigma_{r_{i}}\left(t-\lambda_{k}+\rho\right)-\varphi_{0}\left(\bar{t}_{i}^{\prime}-\lambda_{k}+\rho\right)\right| \leqslant$ $\tau_{i}+B / n_{i}^{3}$. При этом когда $t \in\left[\lambda_{k}-2 \rho, \lambda_{k}\right]$, то $\bar{t}_{i}$ и $\bar{t}_{i}^{\prime} \in[0, \pi]$, если $i$ достаточно велико. А так как в силу (6) и (7)

$$
\begin{aligned}
& \varphi_{0}\left(-t-\lambda_{k}+\rho\right)= 0 \text { и }\left|\sigma_{r_{i}}\left(-t-\lambda_{k}+\rho\right)\right| \leqslant \frac{2 A}{n_{i}^{3}} \text { при } t \in[0, \pi], \\
& \varphi_{k}(t)= \varphi_{0}\left(t-\lambda_{k}+\rho\right) \quad \text { при } t \in[0, \pi], \\
& \varphi_{0}\left(t-\lambda_{k}+\rho\right)= 0 \text { и }\left|\sigma_{r_{i}}\left(t-\lambda_{k}+\rho\right)\right| \leqslant \frac{2 A}{n_{i}^{3}} \\
& \text { при } t \in[0, \pi] \backslash\left[\lambda_{k}-2 \rho, \lambda_{k}\right],
\end{aligned}
$$

TO

$$
\left|\varphi_{k}(t)-S_{r_{i}}(t)\right|=\left|S_{r_{i}}(t)\right| \leqslant \frac{4 A}{n_{i}^{3}} \quad \text { при } t \in[0, \pi] \backslash\left[\lambda_{k}-2 \rho, \lambda_{k}\right] .
$$


И далее с учетом (10) при достаточно больших $i$ получаем

$$
\begin{aligned}
& \left|\varphi_{k}(t)-S_{r_{i}}\left(\bar{t}_{i}\right)\right| \leqslant \tau_{i}+\frac{\Phi}{n_{i}^{3}}, \\
& \left|\varphi_{k}\left(\bar{t}_{i}^{\prime}\right)-S_{r_{i}}(t)\right| \leqslant \tau_{i}+\frac{\Phi}{n_{i}^{3}},
\end{aligned}
$$

где $\Phi=4 A+B$. Неравенства (13) и (14) вместе с неравенствами

$$
\left|t-\bar{t}_{i}\right| \leqslant \alpha \tau_{i}, \quad\left|t-\bar{t}_{i}^{\prime}\right| \leqslant \alpha \tau_{i}
$$

равносильны неравенству $H_{\alpha}\left(\varphi_{k}, S_{r_{i}},[0, \pi]\right) \leqslant \tau_{i}+\Phi / n_{i}^{3}$ для достаточно больших $i$. Ясно также, что

$$
R\left(D\left(\varphi_{k}\right) \cap\left(\lambda_{k}-2 \rho, \lambda_{k}\right)\right) \leqslant\left(2 \pi-2 \alpha \widetilde{c}_{0}\right) /\left(3 \pi-2 \alpha \widetilde{c}_{0}\right) .
$$

Наконец, считаем $b_{k}=\cos \lambda_{k}, \Delta_{k}=\left[b_{k}, b_{k} \cos 2 \rho+\sqrt{1-b_{k}^{2}} \sin 2 \rho\right]$, $\stackrel{\circ}{\Delta}_{k}$-внутренность $\Delta_{k}, g_{k}(x)=\sqrt{1-b_{k}^{2}} \varphi(\arccos x)$ и $Q_{r_{i}}(x)=\sqrt{1-b_{k}^{2}} \times$ $S_{r_{i}}(\arccos x)$ при $x \in \Im$. Функция $S_{r_{i}}(t)$ - полином степени не вьше $r_{i}$ от $\cos t$, а тогда $Q_{r_{i}}(x)$ - алгебраический полином степени не вьше $r_{i}$, для которого

$$
\left|g_{k}(x)-Q_{r_{i}}(x)\right|=\left|Q_{r_{i}}(x)\right| \leqslant \frac{4 A}{n_{i}^{3}} \quad \text { при } \quad x \in \Im \backslash \Delta_{k}
$$

в силу неравенства (12). Пусть $x$ - любое значение из отрезка $\Delta_{k}$ и $g_{k}(x)$ любое значение функции $g_{k}, t=\arccos x$, а $\bar{t}_{i}-$ то значение, при котором выполнены неравенства $(13)$ и $(15)$, когда в них $\varphi_{k}(t)=g_{k}(x) / \sqrt{1-b_{k}^{2}}$. Положив $x_{i}=\cos \bar{t}_{i}$, получим тогда

$$
\begin{gathered}
\left|x-x_{i}\right| \leqslant\left|t-\bar{t}_{i}\right| \cdot\left|\sin \left(\lambda_{k}+\alpha \tau_{i}\right)\right| \leqslant \alpha\left(\sqrt{1-b_{k}^{2}} \tau_{i}+\alpha \tau_{i}^{2}\right), \\
\left|g_{k}(x)-Q_{r_{i}}(x)\right| \leqslant \sqrt{1-b_{k}^{2}} \tau_{i}+\frac{\Phi}{n_{i}^{3}}
\end{gathered}
$$

(при достаточно больших $i$ ). Опираясь на неравенства (14) и (15), аналогичными рассуждениями установим, что для любого $x$ из отрезка $\Delta_{k}$ найдется такое $x_{i}^{\prime}=\cos \bar{t}_{i}^{\prime}$ и некоторое значение $g_{k}\left(x_{i}^{\prime}\right)$ функции $g_{k}$, что

$$
\begin{gathered}
\left|x-x_{i}^{\prime}\right| \leqslant \alpha\left(\sqrt{1-b_{k}^{2}} \tau_{i}+\alpha \tau_{i}^{2}\right), \\
\left|Q_{r_{i}}(x)-g_{k}\left(x_{i}^{\prime}\right)\right| \leqslant \sqrt{1-b_{k}^{2}} \tau_{i}+\frac{\Phi}{n_{i}^{3}} .
\end{gathered}
$$


Неравенства (16)-(19) в совокупности означают, что

$$
H_{\alpha}\left(g_{k}, Q_{r_{i}}, \Im\right) \leqslant \sqrt{1-b_{k}^{2}} \tau_{i}+\frac{L}{n_{i}^{2}},
$$

где $L=2 \max \left\{4 \alpha \widetilde{c}_{0}^{2}, \Phi\right\}$, а $i$ достаточно велико. В силу локальной линейности отображения $x=\cos t(t \in[0, \pi])$ и равенства (11) очевидны соотношения $R\left(D\left(g_{k}\right)\right)=R\left(D\left(\varphi_{k}\right) \cap\left(\lambda_{k}-2 \rho, \lambda_{k}\right)\right) \leqslant\left(2 \pi-2 \alpha \widetilde{c}_{0}\right) /\left(3 \pi-2 \alpha \widetilde{c}_{0}\right)$, где $D\left(g_{k}\right) \subset \stackrel{\circ}{\Delta}_{k}$.

Полученный для алгебраического случая результат удобно резюмировать в виде следующей леммы технического характера.

Лемма $3^{\prime}$. Для любых $\alpha>0, \widetilde{c}_{0} \in(\pi /(2 \alpha), \pi / \alpha), b_{k} \in(0,1)$, любого положительного $\varepsilon_{k}<\arccos b_{k}$ и любой возрастающей последовательности натуральных чисел $\left\{n_{i}^{(0)}\right\}_{i=1}^{\infty}$ существуют заданная на отрезке $\Im$ функиия $g_{k} c \sup _{x \in \Im}\left|g_{k}(x)\right| \leqslant 1$ и последовательность алгебраических полиномов $\left\{Q_{r_{i}}\right\}_{i=1}^{\infty}, r_{i}=n_{i}+m_{i}, m_{i}=\left[\sqrt{n_{i}}+1\right]$, такие, что

1) $H_{\alpha}\left(g_{k}, Q_{r_{i}}, \Im\right) \leqslant\left(\sqrt{1-b_{k}^{2}}\left(\widetilde{c}_{0}+2^{-i+1}\right) / n_{i}\right)+L / n_{i}^{2}(i=1,2, \ldots)$, где $L=$ const, зависящая только от $\alpha$ и $\widetilde{c}_{0}$; причем $g_{k}(x)=0,\left|Q_{r_{i}}(x)\right| \leqslant$ $L / n_{i}^{3}$, ecлu

$$
x \notin \Delta_{k} \stackrel{\text { def }}{=}\left[b_{k}, b_{k} \cos 2 \rho+\sqrt{1-b_{k}^{2}} \sin 2 \rho\right],
$$

где $\rho=\left(2 \alpha \widetilde{c}_{0}-\pi\right) /\left(2 n_{1}\right)<\varepsilon_{k}$;

2) $D\left(g_{k}\right) \subset \stackrel{\circ}{\Delta}_{k}, R\left(D\left(g_{k}\right)\right) \leqslant\left(2 \pi-2 \alpha \widetilde{c}_{0}\right) /\left(3 \pi-2 \alpha \widetilde{c}_{0}\right)$;

3) $\left\{n_{i}\right\}_{i=1}^{\infty}-$ подпоследовательность заданной возрастающей последовательности натуральных чисел $\left\{n_{i}^{(0)}\right\}_{i=1}^{\infty}$, первый член которой может быть взят любым как угодно большим числом.

Чтобы по заданным в теореме 2 числам $\alpha>0, c_{0} \in(0, \pi / \alpha), b \in$ $\left(a\left(c_{0}\right), a^{\prime}\left(c_{0}\right)\right]$ предъявить функцию с требуемыми свойствами, выберем последовательность чисел $\left\{b_{k}\right\}_{k=1}^{\infty}$ из промежутка $\left(a\left(c_{0}\right), a^{\prime}\left(c_{0}\right)\right]$ такую, что $b_{k} \nearrow b$, по индукции введем определенные на отрезке $\Im$ функции $g_{k}(k=1,2, \ldots)$ с $\sup _{x \in \Im}\left|g_{k}(x)\right| \leqslant 1$ и соответствуюшие им последовательности алгебраических полиномов $\left\{Q_{r_{i}^{(k)}}^{(k)}\right\}_{i=1}^{\infty}, r_{i}^{(k)}=n_{i}^{(k)}+m_{i}^{(k)}$, $m_{i}^{(k)}=\left[\sqrt{n_{i}^{(k)}}+1\right]$, со свойствами

$$
\text { 1) } \begin{aligned}
H_{\alpha}\left(g_{k}, Q_{r_{i}^{(k)}}^{(k)}, \Im\right) & \leqslant \frac{c_{0}+2^{-i+1} \sqrt{1-b_{k}^{2}}}{n_{i}^{(k)}}+\frac{L}{\left(n_{i}^{(k)}\right)^{2}} \stackrel{\text { def }}{=} h_{i}^{(k)} \\
& <\frac{\min \left\{b_{k}-b_{k-1}, b_{k+1}-b_{k}\right\}}{2 \alpha} \quad\left(b_{0} \stackrel{\text { def }}{=} a\left(c_{0}\right)\right),
\end{aligned}
$$


где $L=$ const; причем

$$
\begin{aligned}
& g_{k}(x)=0, \quad\left|Q_{r_{i}^{(k)}}^{(k)}(x)\right| \leqslant \frac{L}{\left(n_{i}^{(k)}\right)^{3}}, \\
& \text { если } x \notin \Delta_{k}=\left[b_{k}, b_{k} \cos 2 \rho+\sqrt{1-b_{k}^{2}} \sin 2 \rho\right],
\end{aligned}
$$

где

$$
\rho=\rho_{k}=\frac{\left(2 \alpha c_{0} / \sqrt{1-b_{k}^{2}}\right)-\pi}{2 n_{1}^{(k)}}<\arccos \frac{b_{k+1}+b_{k}}{2}-\arccos b_{k}
$$

2)

$$
D\left(g_{k}\right) \subset \stackrel{\circ}{\Delta}_{k}, \quad R\left(D\left(g_{k}\right)\right) \leqslant \frac{2 \pi \sqrt{1-b_{k}^{2}}-2 \alpha c_{0}}{3 \pi \sqrt{1-b_{k}^{2}}-2 \alpha c_{0}}
$$

3) $\left\{n_{i}^{(k)}\right\}_{i=1}^{\infty}-$ подпоследовательность последовательности $\left\{n_{i}^{(k-1)}\right\}_{i=1}^{\infty}$ $\left(n_{i}^{(0)} \stackrel{\text { def }}{=} i\right)$. Существование функций $g_{k}(k=1,2, \ldots)$ обеспечено леммой $3^{\prime}$. На $k$-м шаге индукции она применяется для заданных чисел $\left.\alpha>0, b_{k} \in(0,1), \widetilde{c}_{0}=c_{0} / \sqrt{1-b_{k}^{2}}, \varepsilon_{k}=\arccos \left(b_{k+1}+b_{k}\right) / 2\right)-\arccos b_{k}$ и определенной на $(k-1)$-м шаге последовательности натуральных чисел $\left\{n_{i}^{(k-1)}\right\}_{i=1}^{\infty}$.

Положим $n_{k}^{(k)}=p_{k}, r_{k}^{(k)}=l_{k}$ и $g(x)=\sum_{k=1}^{\infty} g_{k}(x) / p_{k}^{3}, x \in \Im$. Ясно, что

$$
\begin{gathered}
g(x)=0 \quad \text { при } \quad x \in \Im \backslash \bigcup_{k=1}^{\infty} \Delta_{k}, \\
g(x)=\frac{g_{k}(x)}{p_{k}^{3}} \quad \text { при } \quad x \in \Delta_{k} \quad(k=1,2, \ldots), \\
R(D(g))=\inf _{k} R\left(D\left(g_{k}\right)\right) \leqslant \frac{2 \pi \sqrt{1-b^{2}}-2 \alpha c_{0}}{3 \pi \sqrt{1-b^{2}}-2 \alpha c_{0}} .
\end{gathered}
$$

Покажем, что $c_{\alpha}(g, \Im)=c_{0}$. С этой целью рассмотрим алгебраические полиномы $R_{q}(x) \stackrel{\text { def }}{=} \sum_{k=1}^{q}\left(Q_{l_{q}}^{(k)}(x) / p_{k}^{3}\right)$ степени $\leqslant l_{q}$ и оценим величину $H_{\alpha}\left(g, R_{q}, \Im\right)$. Отметим, что $\Delta_{i} \cap \Delta_{j}=\varnothing$ при $i \neq j$, и что число $p_{q}=n_{q}^{(q)}$ входит во все предыдущие подпоследовательности, однаков каждую со своим порядковым номером, т.е. $n_{q}^{(q)}=n_{s(k)}^{(k)}$ при $k<q$. Очевидно, 
что $s(k) \geqslant q$. Пусть $x$ - любое фиксированное число из отрезка . Если $x \notin \bigcup_{k=1}^{q} \Delta_{k}$, то из $(21)$ и $(22)$ следует

$$
\begin{aligned}
\left|g(x)-R_{q}(x)\right| & \leqslant \sum_{k=1}^{q}\left|g_{k}(x)-Q_{l_{q}}^{(k)}(x)\right|+\sum_{k=q+1}^{\infty}\left(\frac{\left|g_{k}(x)\right|}{p_{k}^{3}}\right) \\
& \leqslant \frac{q L}{p_{q}^{3}}+\frac{1}{p_{q}^{3}} \leqslant \frac{L+1}{p_{q}^{2}} .
\end{aligned}
$$

Если $x \in \Delta_{j}$, где $j$ - одно из чисел $1,2, \ldots, q$, то для любого значения $g_{j}(x)$ функции $g_{j}$ в силу $(20)$ найдется такое $x_{j}$, что $\left|x-x_{j}\right| \leqslant \alpha h_{s(j)}^{(j)}$, $x_{j} \notin \bigcup_{k=1, k \neq j}^{q} \Delta_{k},\left|g_{j}(x)-Q_{l_{q}}^{(j)}\left(x_{j}\right)\right| \leqslant h_{s(j)}^{(j)}$. А тогда, учитьвая $(21)$, получаем

$$
\begin{aligned}
\left|g(x)-R_{q}\left(x_{j}\right)\right| \leqslant & \sum_{k=1, k \neq j}^{q}\left|g_{k}(x)-Q_{l_{q}}^{(k)}\left(x_{j}\right)\right|+\left|g_{j}(x)-Q_{l_{q}}^{(j)}\left(x_{j}\right)\right| \\
& +\sum_{k=q+1}^{\infty} \frac{\left|g_{k}(x)\right|}{p_{k}^{3}} \\
\leqslant & \frac{(q-1) L}{p_{q}^{3}}+h_{s(j)}^{(j)}+\frac{1}{p_{q}^{2}} \leqslant h_{s(j)}^{(j)}+\frac{L+1}{p_{q}^{2}} .
\end{aligned}
$$

С другой стороны, в силу (20), каким бы не был $x \in \Delta_{j}$, найдется такое $x_{j}^{\prime}$ и значение $g_{j}\left(x_{j}^{\prime}\right)$ функции $g_{j}$, что $\left|x-x_{j}^{\prime}\right| \leqslant \alpha h_{s(j)}^{(j)}, x_{j}^{\prime} \notin \bigcup_{k=1, k \neq j}^{q} \Delta_{k}$, $\left|g_{j}\left(x_{j}^{\prime}\right)-Q_{l_{q}}^{(j)}(x)\right| \leqslant h_{s(j)}^{(j)}$. Поэтому

$$
\begin{aligned}
\left|g_{j}\left(x_{j}^{\prime}\right)-R_{q}(x)\right| \leqslant \sum_{k=1, k \neq j}^{q} \mid g_{k}\left(x_{j}^{\prime}\right) & -Q_{l_{q}}^{(k)}(x)|+| g_{j}\left(x_{j}^{\prime}\right)-Q_{l_{q}}^{(j)}(x) \mid \\
& +\sum_{k=q+1}^{\infty} \frac{\left|g_{k}(x)\right|}{p_{q}^{3}} \leqslant h_{s(j)}^{(j)}+\frac{L+1}{p_{q}^{2}} .
\end{aligned}
$$

Неравенства (24)-(26) означают, что

$$
H_{\alpha}\left(g, R_{q}, \Im\right) \leqslant \max _{j=1,2, \ldots, q}\left\{h_{s(j)}^{(j)}\right\}+\frac{L+1}{p_{q}^{2}} .
$$

TaK как $p_{q}=n_{q}^{(q)}=n_{s(j)}^{(j)}, s(j) \geqslant q$,

$$
h_{s(j)}^{(j)} \leqslant \frac{c_{0}+2^{-s(j)+1} \sqrt{1-b_{k}^{2}}}{n_{s(j)}^{(j)}}+\frac{L}{\left(n_{s(j)}^{(j)}\right)^{2}}
$$


TO

$$
\max _{j=1,2, \ldots, q}\left\{h_{s(j)}^{(j)}\right\} \leqslant \frac{c_{0}+2^{-q+1}}{p_{q}}+\frac{L}{p_{q}^{2}} .
$$

Ввиду того, что $l_{q} / p_{q}=\left(p_{q}+\left[\sqrt{p_{q}}+1\right]\right) / p_{q} \rightarrow 1$ при $q \rightarrow \infty$, с учетом последнего неравенства оказьвается

$$
l_{q} H_{\alpha}\left(g, R_{q}, \Im\right) \leqslant \frac{l_{q}\left(c_{0}+2^{-q+1}\right)}{p_{q}}+\frac{l_{q} L}{p_{q}} \rightarrow c_{0} \quad \text { при } \quad q \rightarrow \infty .
$$

Таким образом, $c_{\alpha}(g, \Im)=\underline{\lim } n H_{\alpha} E_{n}(g, \Im) \leqslant c_{0}$. По лемме 1 в согласии с оценкой (23) имеем

$$
\frac{2 \pi \sqrt{1-b^{2}}-2 \alpha c_{\alpha}(g, \Im)}{3 \pi \sqrt{1-b^{2}}-2 \alpha c_{\alpha}(g, \Im)} \leqslant R(D(g)) \leqslant \frac{2 \pi \sqrt{1-b^{2}}-2 \alpha c_{0}}{3 \pi \sqrt{1-b^{2}}-2 \alpha c_{0}}
$$

что возможно только при $c_{\alpha}(g, \Im)=c_{0}$. Теорема 2 доказана.

Восточноукраинский государственный университет

Поступило

29.12 .94

\section{СПИСОК ЦИТИРОВАННОЙ ЛИТЕРАТУРЫ}

[1] Сендов Бл. Хаусдорфовые приближения. София: Изд-во Болг. АН, 1979.

[2] Долженко Е.П., Севастьянов Е.А. О зависимости свойств функций от скорости их приближения полиномами // Изв. АН СССР. Сер. матем. 1978. T. 42. C. $270-304$.

[3] Долженко Е. П., Севастьянов Е. А. О приближении функций в хаусдорфовой метрике // Докл. АН СССР. 1976. Т. 226. № 4. С. 768-770.

[4] Петрушев П., Ташев С. Некоторые обратные теоремы в метрике Хаусдорфа // Докл. Болг. АН. 1976. Т. 29. С. 1721-1724.

[5] Ермаков А. И. Наилучшие хаусдорфовы приближения алгебраическими полиномами и непрерывность функции // Матем. заметки. 1980. Т. 28. №6. C. $843-858$.

[6] Петухов А. П. О зависимости свойств множества точек разрыва функции от скорости ее полиномиальных хаусдорфовых приближений // Матем. сб. 1989. Т. 180. № 7. С. 969-988.

[7] Долженко Е.П. Граничные свойства произвольных функций // Изв. АН СССР. Сер. матем. 1967. Т. 31. № 1. С. 3-14. 